\title{
Comparing the Resynthesis of Brassica napus L. by Interspecific Somatic and Sexual Hybridization. I. Producing and Identifying Hybrids
}

\author{
Richard H. Ozminkowski, Jr., ${ }^{1}$ and Pablo Jourdan ${ }^{2}$ \\ Department of Horticulture, The Ohio State University, 2001 Fyffe Court, Columbus OH 43210-1096
}

\begin{abstract}
Additional index words. disomic polyploids, heterozygosity, polyploidy, protoplast fusion, embryo rescue
Abstract. Brassica napus (genome aacc), a natural allotetraploid derived from hybridization between B. oleracea L. (genome $c c$ ) and $B$. rapa L. (genome $a a$ ), was synthesized by sexual and somatic interspecific hybridizations from the same parent plants to compare the two methods of combining genomes and assess the genetic consequences of bypassing the gametophytic phase before hybrid formation. Highly heterozygous species parents were first produced by intraspecific hybridization between two subspecies each of $B$. oleracea and $B$. rapa. Leaf tissue from young plants of both parental species served as a source of protoplasts for fusion; the same plants were later used for crosses. Seventy-two somatic hybrids were produced using a polyethylene glycol-mediated fusion protocol and 27 sexual hybrids were obtained by embryo rescue. Somatic hybrids were produced between one $B$. oleracea and two sibling $B$. rapa plants. Sexual hybrids were successfully produced with only one of the two $B$. rapa siblings. Hybrids were identified by morphology, isozyme patterns, and total DNA content. Although fertile allotetraploid somatic hybrids were obtained within 7 months after seeding parent lines, >1 year was required to produce fertile sexual hybrids.
\end{abstract}

Brassica napus is an allopolyploid crop of major worldwide importance used primarily as an oilseed, but it is also used in limited areas as a table vegetable ('Hakuran' rutabaga) and as fodder. 'Hakuran' is an artificially synthesized form of this species developed in Japan by interspecific sexual hybridization of Chinese cabbage (B. rapa, Pekinensis Group, syn. B. campestris cv. Hakusai) and cabbage (B. oleracea Capitata Group, cv. Kanran) as a new vegetable that combined the heading characteristics of both parents (Nishi, 1980). Given the large morphological variability present in the two progenitor species, entirely new vegetable forms of $B$. napus may be developed by exploiting the morphological plasticity of these species. Interspecific hybridization of $B$. rapa and $B$. oleracea to synthesize $B$. napus can be achieved by sexual crosses between diploid or tetraploid parents (Inomata, 1978; Olsson, 1960) and protoplast fusion of diploid parents (Jourdan et al., 1989; Sundberg and Glimelius, 1986; Terada et al., 1987). This possibility permits the examination of two very different methods of combining genomes: one dependent on the generative cycle and the other based on somatic cells.

The types of gametes involved in sexual hybridization lead to different outcomes with potentially significant long-term consequences. Hybridization by reduced $(n)$ gametes from diploid $(2 x)$ parents followed by doubling of the hybrid essentially fixes homozygosity within each genome; overall heterozygosity is increased by using reduced gametes of $4 x$ parents and increased further by $2 n$ gametes — via first or second division restitution —of $2 x$ parents (Peloquin and Ortiz, 1992; Sanford, 1983; Sybenga, 1983). Heterozygosity would be maximized by using somatic cells

Received for publication 19 May 1992. Accepted for publication (pending acceptance of part 2) 29 Sept. 1993. Salaries and research support provided by state and federal funds appropriated to the Ohio Agricultural Research and Development Center, The Ohio State Univ. Manuscript no. 141-92. Use of trade names does not imply endorsement of the products named nor criticism of similar ones omitted. The cost of publishing this paper was defrayed in part by the payment of page charges. Under postal regulations, this paper therefore must be hereby marked advertisement solely to indicate this fact.

'Present address: Heinz U.S.A., 13737 Middleton Pike, Bowling Green, OH 43402-9481.

${ }^{2}$ To whom reprint requests should be addressed.
(Sanford, 1983). Disomic polyploids synthesized by combining reduced gametes from diploid species fix intragenomic homozygosity but intergenomic heterozygosity by the presence of two species genomes. In contrast, somatic hybrids should combine intra- and intergenomic heterozygosity. Whether this high degree of heterozygosity in somatic hybrids translates into any advantage for a germplasm enhancement strategy in a disomic polyploid such as $B$. napus has not been carefully examined. Availability of plants produced by sexual and somatic hybridization would allow such examination.

Surprisingly little information exists on the comparison of somatic and sexual hybrids. We believe that this paucity exists primarily because most somatic hybridizations initially focused on combinations of species difficult or impossible to achieve by sexual hybridization, but perhaps also because the comparison is believed by some to be intuitively obvious. Recent work on the manipulation of cytoplasmic genomes and in the transfer of parts of the nuclear genome by asymmetric hybridization has been emphasized (Kumar and Cocking, 1987; Negrutiu et al., 1989). There are considerable mechanistic differences between the two hybridization methods - meiosis and haploid gamete competition in sexual hybridization vs. mitosis and nonselective diploid fusion in somatic hybridization - that should translate into potentially significant genetic differences. In addition, the biological factors limiting the production of the interspecific hybrids differ. Sexual hybridization is limited by various interactions between cells in the flowers, whereas interactions within cells limit the success of somatic hybridization (Harms, 1983). While the two hybridization systems have been compared conceptually (Glimelius, 1985; Harms, 1983; Negrutiu et al., 1989; Sundberg et al., 1987; Sybenga, 1983), there have been no direct empirical evaluations of hybrids derived by the two methods.

Protoplast fusion may offer several potential advantages over sexual crosses in hybrid production, including novel cytoplasmic combinations and retention of parental heterozygosity; hybridization of a sterile plant that may have desirable attributes; opportunity for potential chromosomal associations or modification of chromosomal constitutions while in vitro; possibility of overcoming sexual incongruity barriers; and possibility of maintaining 
desirable gene combinations, especially if heterozygous and quantitative (Glimelius, 1985; Negrutiu et al., 1989). However, past experience with somatic hybrids shows that these advantages have not been fully realized and that there are constraints still to be addressed with protoplast fusion (Negrutiu et al., 1989). Resynthesis of $B$. napus provides an opportunity to carefully evaluate some of these issues. Such resynthesis is more than an academic exercise. It is desirable at various levels of a genetic improvement program depending on the specific use of the crop (i.e., as oilseed, vegetable, or fodder) and breeders' goals. Some goals for resynthesis include 1) broadening the germplasm base-of nuclear, chloroplast, or mitochondrial genes - to include much of the variability in the progenitor species (Chen and Heneen, 1989; Kräling, 1987); 2) studying the process of diploidization by comparing newly synthetic plants with the natural crop and evaluating such processes as genome silencing; and 3) introducing specific characteristics of the progenitors (e.g., yellow seeds). As Chen and Heneen (1989) stated, "...resynthesized lines of [B. napus] are definitely not a simple addition of the parental genetic potential. The prevalence of various interactions between the two parental genomes gives rise to novel variations which may be of practical interest." Here we describe and assess a strategy for interspecific hybridization in which the same plants are used for protoplast fusion and sexual hybridization at the diploid level. In a second report, we describe the hybrids in more detail (Ozminkowski and Jourdan, 1993b).

\section{Materials and Methods}

Plant material. The B. oleracea species parent was derived from the cross 'Blue Diamond' cauliflower [Botrytis Group, Harris-Moran, Salinas, Calif.] x purple ornamental kale, (Acephala Group; Northrup King, Minneapolis). The B. rapa (Rapifera Group) species parent was a hybrid of 'Purple Top White Globe' turnip (Harris-Moran) X 'Michihili' chinese cabbage, (Pekinensis Group; Harris-Moran). Seeds of both species parents were surfacesterilized [1.6\% sodium hypochlorite for $20 \mathrm{~min}$, three rinses in sterile distilled water $\left.\left(\mathrm{sdH}_{2} \mathrm{O}\right)\right]$ and grown on Murashige and Skoog (MS) medium (Murashige and Skoog, 1962) [MS salts, Gamborg's B5 vitamins, 3\% sucrose, $\mathrm{pH}$ 5.8, 0.8\% Sigma (St. Louis) agar]. A single B. oleracea seedling (-2) and two B. rapa seedlings ( -3 and -4$)$ were propagated by shoot culture on $\mathrm{MS}_{0}$ to produce multiple clones. Clones of each species parent were transferred to soil and grown in a growth chamber for use as protoplast sources. After fusion experiments, species parent plants were transferred to the greenhouse and grown to flowering for sexual hybridization. Both $B$. rapa siblings were vernalized (4C, $24 \mathrm{~h}$ light, 4 weeks) to enhance flowering.

Producing somatic hybrids. Leaf tissue from young, growthchamber-grown plants was surface-sterilized (90 sec in 70\% ethanol, 6 min in $0.5 \%$ sodium hypochlorite, three rinses in $\mathrm{sdH}_{2} \mathrm{O}$ ) and protoplasts were isolated as described by Robertson et al. (1987). Protoplasts of B. oleracea were inactivated with filtersterilized $2.3 \mathrm{~mm}$ iodoacetic acid for $\approx 25 \mathrm{~min}$ before fusion. In the first of two fusion experiments, $B$. oleracea-2 protoplasts were combined with those of $B$. rapa-4. The fusion procedure was based on that of Robertson et al. (1987), except that polyethylene glycol 1500 (Aldrich, Milwaukee) was used and the elution solution consisted of $500 \mathrm{~mm}$ sorbitol and $50 \mathrm{~mm} \mathrm{CaCl}_{2} \cdot 2 \mathrm{H}_{2} \mathrm{O}, \mathrm{pH}$ 8.0. The second experiment was similar to the first except that $B$. oleracea2 protoplasts were fused to those of $B$. rapa-3. Dimethylsulfoxide $(10 \% \mathrm{v} / \mathrm{v})$ was added to the above fusion solution; this fusion was carried out in a petri plate, similar to the protocol of Jourdan et al. (1989). Protoplasts in both experiments were plated at an initial density of $2.0 \times 10^{5} / \mathrm{ml}$ medium B (Pelletier et al., 1983), without Tween 80, in each well of 12-well polystyrene plates (Corning, Corning, N.Y.) and cultured on media E and F as described by Jourdan and Earle (1989). The concentrations of growth regulators in medium $E$ were adjusted to $5.4 \mu_{\mathrm{M}} \alpha$-naphthalene acetic acid, $2.2 \mu \mathrm{M}$ 6-benzylaminopurine (BA), and $2.5 \mu \mathrm{M} \mathrm{N} \mathrm{N}^{6}$-isopentyl adenine. Calluses with shoot primordia were transferred to Medium $\mathrm{F}$ and, once established, grown on $\mathrm{MS}_{0}$. Untreated control protoplasts of the species parents were cultured in the same media.

Producing sexual hybrids. Reciprocal pollinations were made between the same $B$. olerace $a$ and $B$. rapa plants used for somatic hybridization using emasculated flower buds at $\approx 2$ days before anthesis. The crosses were made during November and December under natural lighting in the greenhouse. Thirteen to 18 days after pollination, siliques were collected, soaked in distilled water for 15 to $30 \mathrm{~min}$ and surface-sterilized ( $1 \mathrm{~min} 70 \%$ ethanol, $5 \mathrm{~min} 0.5 \%$ sodium hypochlorite, three rinses in $\mathrm{sdH}_{2} \mathrm{O}$ ). Ovules were removed from siliques under aseptic conditions on a moist paper towel, and the distal end of the ovule (opposite the chalaza) was cut off. Dissected ovules were placed in the liquid medium of Quazi (1988), from which tri-potassium phosphate was omitted, or on the liquid medium of Ripley and Arnison (1990). Cultures were placed in $\operatorname{dim} \operatorname{light}\left(60 \mu \mathrm{mol} \cdot \mathrm{m}^{-2} \cdot \mathrm{s}^{-1}\right)$ on a gyratory shaker $(\approx 35 \mathrm{rpm})$ until embryos developed to the torpedo stage ( 2 to 3 weeks). Embryos were transferred to B5G1 shoot development medium (Ripley and Arnison, 1990), solidified with $0.8 \%$ washed agar (Sigma), and grown at 4C with an 8-h photoperiod for 10 days. Shoots were multiplied on solid $\mathrm{MS}_{0}$ containing $0.9 \mu \mathrm{M} \mathrm{BA}$, and normal shoots were subsequently maintained on $\mathrm{MS}_{0}$.

Chromosome doubling of sexual hybrids. Because the sexual hybrids could be sterile allodiploids, a step to induce chromosome doubling was included in this procedure. Clones of 10 sexual hybrids were treated with colchicine; in vitro shoots (128 explants in total) were placed in $\mathrm{MS}_{0}$ containing $0.05 \%$ (w/v) colchicine (added to autoclaved medium from a filter-sterilized $5 \%$ solution). Five or 7 days later, explants were transferred to $\mathrm{MS}_{0}$ lacking colchicine and cultured for 2 months until the survivors were placed in soil. After 3 weeks in soil, plants were vernalized as above, then grown in the greenhouse. One $F_{1}$ hybrid (\#7) became fertile after plant regeneration from leaf protoplasts, probably through spontaneous doubling during the cell culture phase.

Isozyme analysis. Putative hybrids were analyzed for glucosephosphate isomerase (GPI, E.C. 5.3.1.9), phosphoglucomutase (PGM, E.C. 2.7.5.1), and shikimate dehydrogenase (SKDH, E.C. 1.1.1.25) in starch gel or cellulose-acetate electrophoresis systems. Leaf tissue from in vitro- or greenhouse-grown material was ground on ice either in $100 \mathrm{~mm}$ tris or $50 \mathrm{~mm}$ glutathione, $\mathrm{pH}$ 8.0, and run on the starch gel system I of Gottlieb (1981) or in the grinding buffer of Hebert and Beaton (1989) (100 mm tris $\mathrm{HCl}, \mathrm{pH}$ 8.0; $64 \mathrm{~mm} \beta$-mercaptoethanol; $0.3 \mathrm{~mm}$ PVP-40T; $0.7 \mathrm{~mm} \beta$ $\mathrm{NADP}^{+}$) for cellulose acetate (Helena Laboratories, Beaumont, Texas). In the latter system, gels were first soaked in $30 \mathrm{~mm}$ tris and $190 \mathrm{~mm}$ glycine, $\mathrm{pH} \mathrm{8.2,} \mathrm{and} \mathrm{run} \mathrm{in} \mathrm{the} \mathrm{same} \mathrm{buffer} \mathrm{for} 20 \mathrm{~min}$ at $200 \mathrm{~V}$. Enzymes were visualized by staining in substrate solution for starch gels or by an agar overlay of the same reaction mixtures for cellulose acetate. Substrate solutions were as described by Thorpe et al. (1987), except that twice the amount of $\beta$-NAD+ replaced $\beta-\mathrm{NADP}^{+}$in the GPI assay mixture and the PGM assay contained twice the indicated amount of glucose-1-phosphate.

Determining ploidy. Ploidy was determined on young vegetative tissue by flow cytometry procedures modified from Arumuganathan and Earle (1991). About $1.5 \mathrm{~g}$ of leaf tissue from greenhouse-grown plants was finely chopped on ice in $10 \mathrm{ml}$ buffer 
to release nuclei. The crude extract was filtered through four layers of cheesecloth and one layer of Miracloth (Calbiochem, La Jolla, Calif.) and then centrifuged at $13 \times g$ for $15 \mathrm{~min}$. The pellet was resuspended gently in $1 \mathrm{ml}$ buffer, and $1 \mu \mathrm{g}$ RNAse-A was added. After $15 \mathrm{~min}$ at room temperature, $20 \mu \mathrm{l}$ propidium iodide (PI) solution $\left(5 \mathrm{mg} \cdot \mathrm{ml}^{-1}\right)$ was added. Samples were analyzed on a flow cytometer (Epics Elite; Coulter, Miami). The intensity of PI estimates DNA content relative to a standard. We used B. napus 'Westar', reported to contain 2.34 pg DNA per diploid nucleus (the 2C value; Arumuganathan and Earle, 1991), as the standard. Chromosome numbers were estimated for 16 somatic hybrids and one clone of 10 sexual hybrids. Buds were fixed (3 ethanol : 1 glacial acetic acid) and pollen mother cells were stained with saturated acetocarmine.

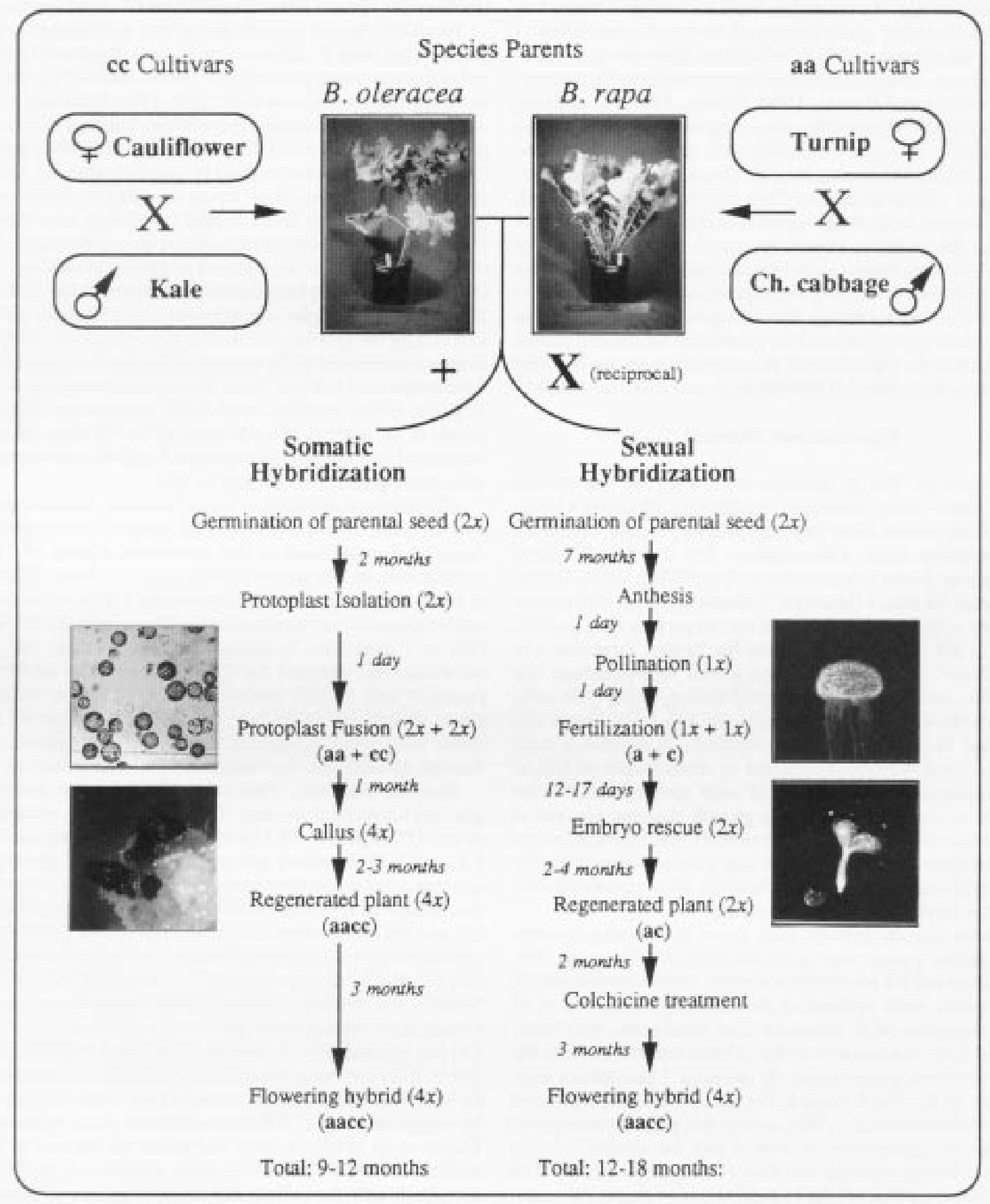

Fig. 1. Strategy for producing somatic and sexual hybrids from highly heterozygous species parents, and time scale for each hybridization. The pistillate and pollen parent for each cultivar is indicated; $a a$ and $c c$ are the genome descriptors for Brassica rapa and B. oleracea, respectively. Photographs to the left of the somatic hybridization column show isolated protoplasts and a regenerated shoot on a callus. Photographs to the right of the sexual hybridization column show a fluorescence micrograph of a stigma covered with germinated pollen grains and an interspecific embryo. 


\section{Results}

Species parents. In the first part of our study, we produced highly heterozygous lines of the progenitor species by crossing diverse cultivars to provide potentially extensive variation in the resynthesized B. napus populations. A summary of the overall strategy is illustrated in Fig. 1. Our use of heterozygous species parents parallels the strategy used by McNaughton and Ross (1978) in the sexual resynthesis of B. napus. Reciprocal crosses were initially made between $B$. oleracea cultivars of ornamental kale, cauliflower, and broccoli. All crosses were successful, and seedlings were germinated in vitro to subsequently isolate protoplasts and evaluate cell culture and plant regeneration characteristics. We had previously identified the kale cultivar as one that exhibited very efficient plant regeneration from leaf protoplasts. Efficient regenerability of one parent involved in fusion is important for producing somatic hybrids (Jourdan et al., 1989). A similar crossing scheme was followed with the $B$. rapa cultivars of turnip and Chinese cabbage. However, plants could not be routinely regenerated from leaf protoplasts of either cultivar. Each seedling derived from the intraspecific crosses was numbered, and we chose to use $B$. oleracea-2, derived from a cauliflower $x$ kale cross, and $B$. rapa -3 and -4 , derived from a turnip $\mathrm{x}$ chinese cabbage cross, for interspecific hybridization. We refer to these plants as species parents.

The species parents differed in many morphological and physiological characteristics inherited from the cultivars, including their ability to regenerate shoots from leaf protoplasts. Control cultures of $B$. oleracea- 2 protoplasts regenerated whole plants at frequencies similar to those of the kale parent, whereas control cultures of B. rapa-3 and - 4 protoplasts formed calluses, but plants could not be regenerated in our culture medium (unpublished observations). The $B$. oleracea- 2 species parent grew vigorously $(>1.7 \mathrm{~m}$ at anthesis) and exhibited morphological traits of each parental cultivar such as waxy leaves and white flowers (Ozminkowski and Jourdan, 1993b). Abundant fruit and seed were produced on these plants in the greenhouse without the need for pollination, a result indicating that the plants were self-compatible (Ozminkowski and Jourdan, 1993a). The two B. rapa species parents also grew vigorously ( $1 \mathrm{~m}$ at anthesis) and were morpho-

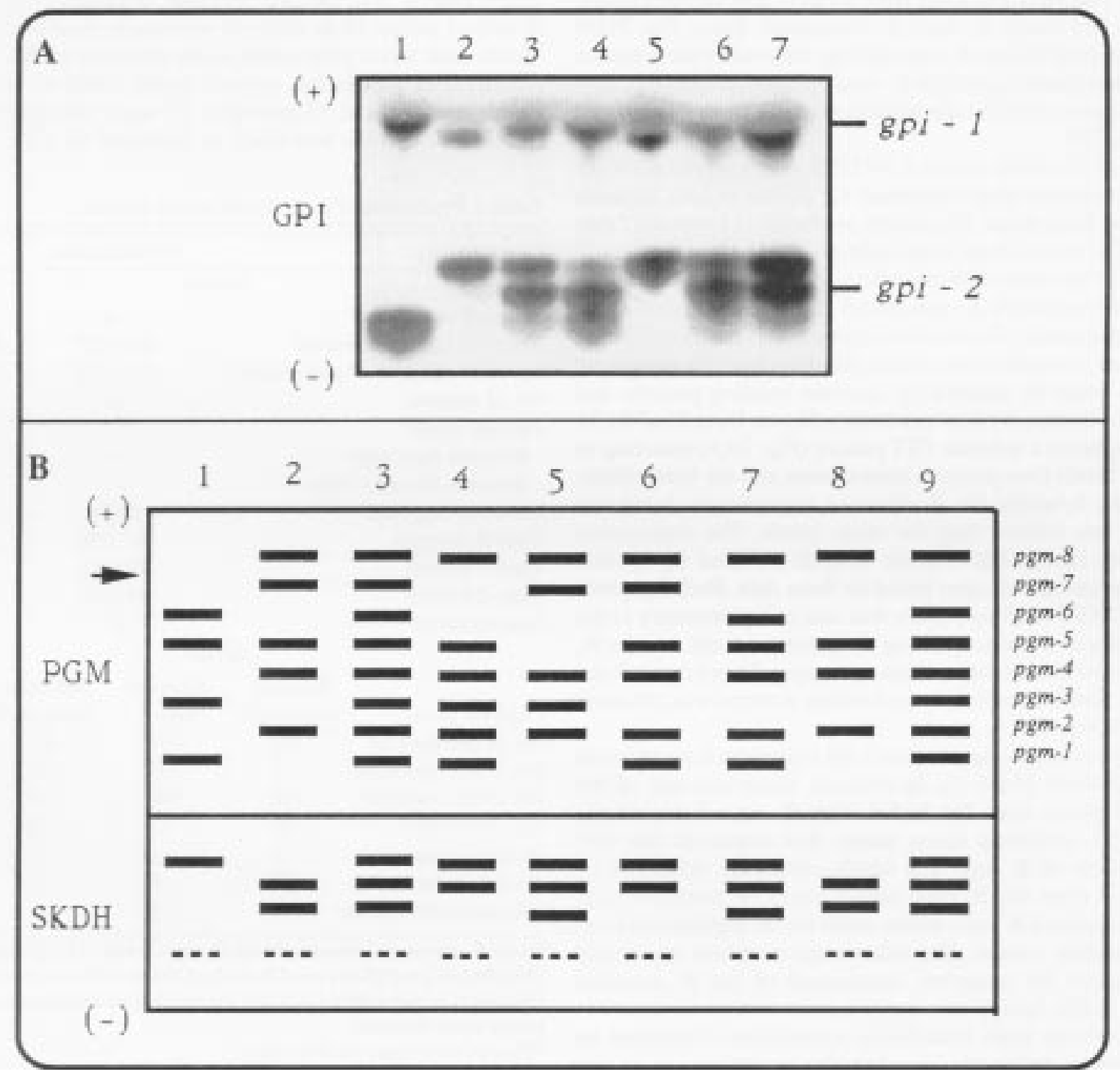

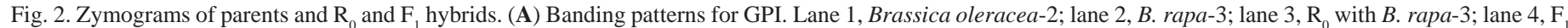

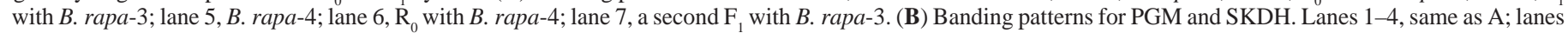

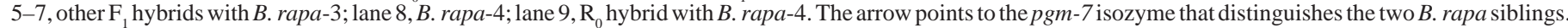


logically indistinguishable from each other. These plants were generally difficult to grow under our greenhouse conditions; they were susceptible to environmental stresses and pests (aphids, Fusarium spp., powdery mildew, turnip mosaic virus), which eventually delayed the production of sexual interspecific hybrids. The initial four clones (not vernalized) died after 6 months in soil, before pollinations could be made; a second pair of clones was brought to flower 3 months after it was put in soil. The yellow flowers of both plants would set no fruit in the greenhouse unless they were cross-pollinated or bud-selfed, a result indicating that these plants were self-incompatible (Ozminkowski and Jourdan, 1993a).

The two species parents could also be distinguished based on GPI, PGM, and SKDH banding patterns. Gpi-2 was uniform within each species, despite the diversity of the cultivar parents, yet variable between species (Fig. 2A). Gpi-1 was monomorphic between the two species parents when resolved in starch gel electrophoresis but apparently dimorphic when resolved in cellulose acetate (not shown); however, variability in resolution between electrophoretic runs precluded using gpi-1 as a distinguishing marker. The SKDH banding patterns were also uniform within the species and could be used to distinguish them. The PGM banding pattern of the two $B$. rapa siblings differed by the presence of an additional band (pgm-7) in B. rapa-3 (lanes 2 and 8, Fig. 2B), but both patterns could be distinguished easily from $B$. oleracea (lane 1, Fig. 2B).

Somatic $\left(R_{0}\right)$ hybrids. About $4.3 \times 10^{6}$ B. oleracea and $6.2 \times 10^{6}$ $B$. rapa protoplasts were combined for fusion in two separate experiments; from these, 58 calluses produced 111 shoots (Table 1, Fig. 1). The shoots from seven calluses died before they could be analyzed. The major difference between the two fusion experiments was in the specific $B$. rapa parent (either -3 or -4 ) used as the source of protoplasts. Shoots were regenerated rapidly following fusion so that, 4 months after culture, plantlets from the remaining 51 calluses could be assayed for isozyme banding patterns and nuclear DNA content by flow cytometry. Shoots from 42 of the 51 calluses displayed a uniform GPI pattern (Fig. 2A) consisting of three gpi-2 bands (two parental homodimers and the heterodimer unique to the hybrids); the $B$. oleracea homodimeric band was commonly less intense than the other bands. The regenerated plants were classified as somatic hybrids (labeled $\mathrm{R}_{0}$ hybrids hereafter) or parental escapes based on these data. Each $\mathrm{R}_{0}$ hybrid displayed a PGM banding pattern that was complementary to the unique parents and uniform among all fusion products, i.e., the $\mathrm{R}_{0}$ hybrids derived from $B$. rapa-4 lacked the $p g m-7$ band (lanes 3 and 9, Fig. 2B). Similar uniformity in banding patterns was obtained with SKDH (Fig. 2B).

In the fusion involving $B$. rapa-3, all regenerated plants were pubescent hybrids (Table 1). In contrast, about one-half of the regenerated plants from the fusion with $B$. rapa-4 was of the parental type, including seven plants that displayed the GPI banding pattern of $B$. rapa and which eventually could not be distinguished from the $B$. rapa parent based on morphological characteristics; such $B$. rapa plants could not be regenerated from control protoplast culture. The iodoacetate treatment apparently was insufficient for complete inactivation of the $B$. oleracea protoplasts in this fusion.

The $\mathrm{R}_{0}$ hybrids from both fusion experiments displayed an overall intermediate morphology, but all were pubescent, as was the $B$. rapa parent. Growth habit was slightly variable, but the plants could be differentiated into two distinct groups. The first group (type-1 hybrids) consisted of a uniform population of highly vigorous plants that displayed normal, symmetrical features. The second group (type-2 hybrids) consisted of a more variable population of less-vigorous plants having rugose and asymmetrical leaves. These morphological features were directly correlated with the ploidy level of the plants. Six type-1 hybrids evaluated exhibited a nuclear DNA content within $15 \%$ of 'Westar' (2.2 to $2.6 \mathrm{pg}$ ), a content that is about the sum of the two species parents $(1.3 \mathrm{pg}$ for B. olerace a and $1.0 \mathrm{pg}$ for B. rapa ) and indicates allotetraploidy (Fig. 3C). Ten type-2 hybrids evaluated had variable DNA content, 3.5 to $5.7 \mathrm{pg}$, but all had a higher DNA content than 'Westar', a result indicative of higher ploidy levels (i.e., hexaploid or octaploid; Fig. 3D). Chromosome counts of 11 other $\mathrm{R}_{0}$ hybrids confirmed that type-2 plants were not simply aneuploids but were of significantly higher ploidy, whereas type- 1 plants were tetraploid $(n=2 x$ = 19; unpublished observations).

Sexual $\left(F_{1}\right)$ hybrids. From $\approx 800$ pollinations, 33 embryos developed into plants, i.e., parental types or $F_{1}$ plants (Table 1). Excised ovules initially cultured only on the modified medium of Quazi (1988) produced viable embryos; culture of entire ovaries was unsuccessful. None of the embryos (Fig. 1) germinated directly into a single plant; instead, they developed into abnormally shaped and swollen leaf-like organized masses mostly devoid of callus. Most embryos eventually produced 20 or more shoots each when propagated in the presence of BA. Three to 5 months after pollination, normal shoots could be examined for isoenzymes. Of the 33 embryos, 27 were interspecific hybrids (labeled $\mathrm{F}_{1}$ hybrids hereafter) as indicated by GPI, PGM, and

Table 1. Production of somatic and sexual hybrids.

\begin{tabular}{|c|c|c|}
\hline Characteristic & \multicolumn{2}{|c|}{ Hybridization } \\
\hline \multicolumn{3}{|c|}{ Somatic } \\
\hline Brassica rapa parent $^{\mathrm{z}}$ & -4 & \\
\hline No. of $B$. rapa protoplasts ${ }^{y}$ & $1.6 \times 10^{6}$ & $4.6 \times 10^{6}$ \\
\hline No. of $B$. oleracea -2 protoplasts & $1.6 \times 10^{6}$ & $2.7 \times 10^{6}$ \\
\hline No. of calluses & 32 & 26 \\
\hline Parental plants ${ }^{\mathrm{x}}$ & $0(0)$ & $27(9)$ \\
\hline Brassica rapa -type & --- & $7(2)$ \\
\hline Brassica oleracea -type & --- & $20(7)$ \\
\hline Unknown $\mathrm{R}_{0}$ plants & $3(3)$ & $4(4)$ \\
\hline Total $\mathrm{R}_{0}$ hybrids & $54^{\mathrm{w}}(29)$ & $23(13)^{\mathrm{v}}$ \\
\hline Type-1 hybrids & $20(11)$ & $11(7)$ \\
\hline Type- 2 hybrids & $18(13)$ & $9(7)$ \\
\hline Undetermined & 11 & 3 \\
\hline
\end{tabular}

\begin{tabular}{|c|c|c|c|c|}
\hline & & cual & & \\
\hline Pistillate parent $^{\mathrm{u}}$ & $\begin{array}{c}\text { Brassica } \\
\text { oleracea-2 }\end{array}$ & $\begin{array}{c}\text { Brassica } \\
\text { rapa-3 }\end{array}$ & $\begin{array}{c}\text { Brassica } \\
\text { oleracea-2 }\end{array}$ & $\begin{array}{c}\text { Brassica } \\
\text { rapa-4 }\end{array}$ \\
\hline No. of pollinations & 143 & 407 & 45 & 196 \\
\hline No. siliqua produced & 11 & 106 & 1 & 70 \\
\hline No. ovules cultured & 110 & 360 & 4 & 230 \\
\hline No. of embryos & 40 & 13 & 0 & 4 \\
\hline Parental types & 2 & 1 & --- & 3 \\
\hline Total $F_{1}$ hybrids & 19 & 8 & --- & 0 \\
\hline Allotetraploid hybrids & 6 & 3 & --- & --- \\
\hline
\end{tabular}

${ }^{\mathrm{z}}$ Only $B$. oleracea-2 was used with $B$. rapa -3 and -4 for protoplast fusion. ${ }^{\mathrm{y}}$ Number of protoplasts used in each of the two fusion experiments.

${ }^{x}$ Numbers in parenthesis indicate the number of calluses from which the plants were obtained.

${ }^{\mathrm{w}}$ Five of these later died in vitro.

${ }^{v}$ One callus produced shoots of both types.

uSexual hybridization involved reciprocal crosses between the same combination of plants used for fusion.

${ }^{t} \mathrm{~F}_{1}$ hybrids that had their chromosome number doubled either by colchicine treatment or by protoplast culture. 
SKDH banding patterns (lanes 4 and 7, Fig. 2A; lanes 4-7, Fig. 2B) whereas the remaining were maternal types. These matromorphous plants may have arisen either by pseudogamy, a common occurrence in interspecific Brassica crosses (Olsson, 1960), or from fertilization with contaminating pollen of the pistillate parent. We did not determine the origin of these matromorphs. Sexual hybrids uniformly displayed the same GPI banding pattern seen in the somatic hybrids. More hybrids were obtained when $B$. oleracea was the pistillate parent in crosses with $B$. rapa-3; however, silique development favored the reciprocal cross (Table 1). The cross with $B$. oleracea as pistillate parent was nine times more efficient than the reciprocal, since $18 \%$ of the cultured ovules developed hybrid embryos, whereas only $2 \%$ did so when $B$. rapa-3 was the pistillate parent. No hybrid plants were obtained with $B$. rapa-4 as either parent.

All $\mathrm{F}_{1}$ hybrids were pubescent, as were the $\mathrm{R}_{0}$ hybrids. However, a greater diversity in morphology, particularly leaf shape, was observed among sexual than somatic hybrids (Ozminkowski and Jourdan, 1993b). All F hybrids had nuclear DNA contents about one-half of 'Westar', a result indicating that each had a single copy of the progenitor's genomes (Fig. 3F). When grown to maturity, these plants produced small flowers having small anthers devoid of pollen, a further indication of monoploidy. Metaphase I chromosome preparations of the allodiploid hybrids revealed 19 chromosomes showing considerable pairing and chiasma formation. Four bivalents and one univalent were commonly observed, while the remaining 10 chromosomes formed complex structures. In most cases, each microspore contained nine or 10 chromosomes after anaphase II. Such homeologous pairing was not obvious in $\mathrm{R}_{0}$ hybrids. A clone of an $\mathrm{F}_{1}$ plant was found to have DNA content similar to that of 'Westar' and thicker foliage than the other $F_{1}$ hybrids. This plant had good pollen shed and high pollen stainability ( $87 \%$ with acetocarmine), yet no seed was obtained after 80 bud and open-flower self-pollinations; seed developed when 'Westar' pollen was used. Another clone from the same embryo was allodiploid, a result indicating that the tetraploidization had occurred during culture and was not due to fertilization of diploid gametes.

Chromosome doubling of $F_{1}$ hybrids. Ten $\mathrm{F}_{1}$ hybrids, selected at random, were exposed to colchicine and, of the 128 explants treated, 64 survived to be placed into soil. On average, $37 \%$ of the explants from eight of the 10 hybrids was successfully doubled (i.e., became allotetraploid), as determined by flow cytometric analysis of nuclear DNA content, which was within $10 \%$ of 'Westar' (Fig. 3G). The remaining plants were chimeric for ploidy (9\%; Fig. 3H), of ploidy levels higher than tetraploid $(9 \%)$, or allodiploid (20\%); 5\% was intermediate between diploid and tetraploid. DNA content could not be determined in the remaining $20 \%$ of the plants; all but one of these had rugose leavesindicative of $4 x$ or greater ploidy, which may have accounted for the poor sample quality.

\section{Discussion}

We have successfully resynthesized $B$. napus by somatic and sexual hybridization of the same parents to begin a detailed comparison of characteristics that may be influenced by hybridization mode. Our strategy for comparison included producing highly heterozygous species parents that combined diverse genotypes that could potentially maximize differences between intragenomic homozygous $\left(\mathrm{F}_{1}\right)$ and heterozygous $\left(\mathrm{R}_{0}\right)$ hybrids derived from the same plants. We selected genotypes primarily based on morphology, evolutionary relationships, and in vitro culture characteris- tics. Each cultivar represents a different morphological modification: leaf shape and color in kale, enlarged immature flower heads in cauliflower, swollen hypocotyl in turnip, and heading in Chinese cabbage. Kale and turnips are derived from the more primitive subspecies of the parental species, whereas cauliflower and Chinese cabbage are more modern subspecies (Song et al., 1988). Kale had a dominant trait of efficient plant regeneration from protoplasts. All of these characteristics are important because producing interspecific hybrids depends on many factors, but paramount among them is the parents' genotypes (Hadley and Openshaw, 1980).

We applied established and straightforward cell culture technologies to produce hybrids. We confirmed that somatic synthesis can work well, provided one parent regenerates shoots from leaf protoplasts at reasonably high frequencies; this is common among many B. oleracea genotypes (Jourdan and Earle, 1989). Inactivation of the highly regenerable parent with a metabolic inhibitor such as iodoacetic acid or Rhodamine-6-G is coupled to the rescue

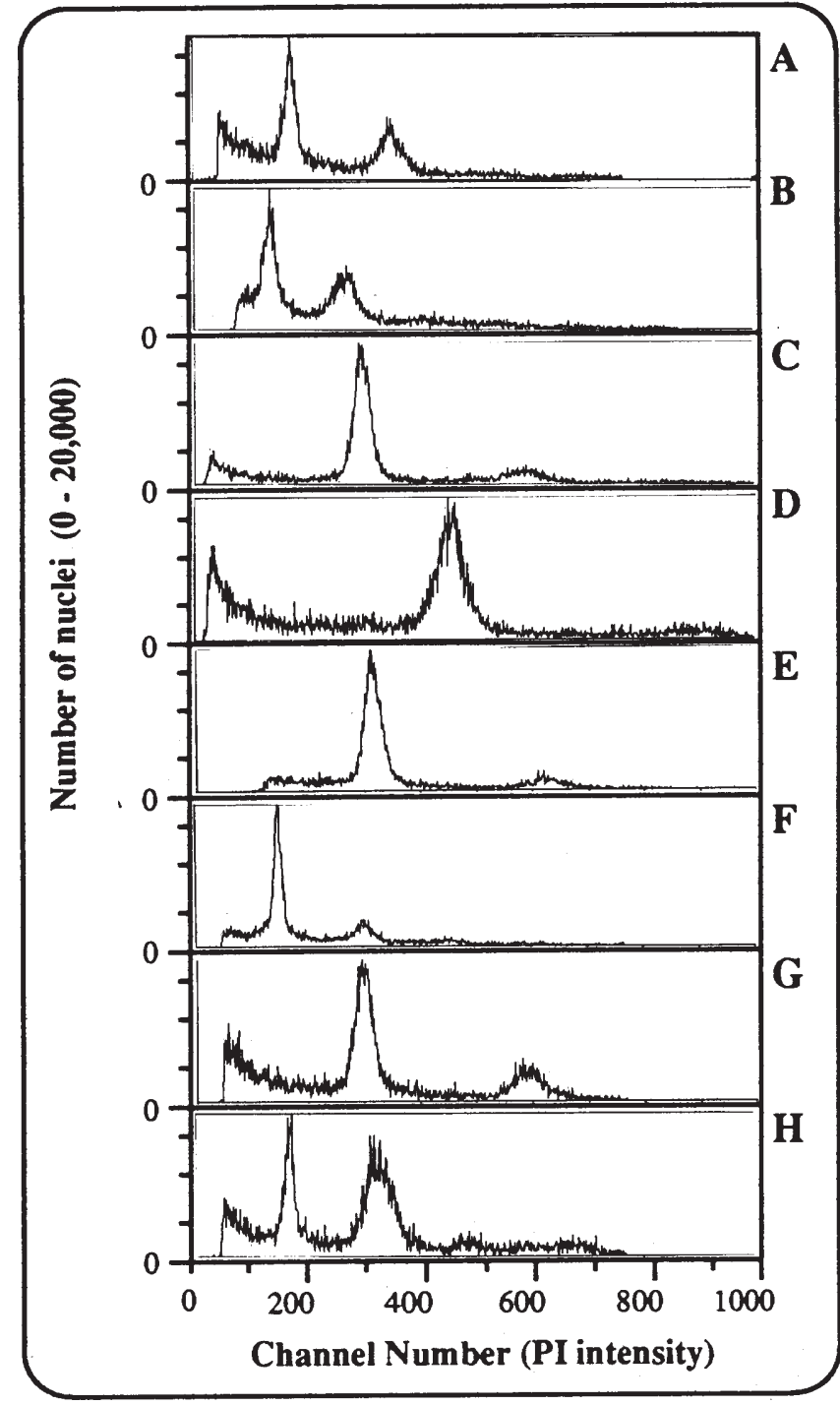

Fig. 3. Histograms of propidium iodide intensity in stained nuclei isolated from (A) Brassica oleracea-2; (B) B. rapa-4; (C) type-1 $\mathrm{R}_{0}$ hybrid; (D) type-2 $\mathrm{R}_{0}$ hybrid; (E) B. napus 'Westar'; (F) an allodiploid $(2 x) \mathrm{F}_{1}$ hybrid; (G) the allotetraploid ( $4 x$, colchicine-doubled) $\mathrm{F}_{1}$ hybrid in $\mathrm{F} ;(\mathbf{H}) \mathrm{F}_{1}$ hybrid showing $2 x$ and $4 x$ nuclei in the same extract. The $y$ axis represents counts of individual nuclei that were detected by fluorescence for each preparation; the $\mathrm{x}$ axis indicates the channel number in the flow cytometer and corresponds to fluorescence intensity. 
of hybrid cells by complementation with metabolically active protoplasts of the other parent which, as is typical among B. rapa genotypes, can be quite recalcitrant to protoplast culture (Terada et al., 1987). Although this approach increases the proportion of somatic hybrids found among the regenerated plants, escapes can occur; in one experiment, nine of the 22 calluses that regenerated shoots were parental plants. Some of these parental escapes were $B$. rapa plants, which we have been unable to regenerate into plants from control cultures. This phenomenon has been observed previously (Jourdan et al., 1989) and its basis is not known. We also confirmed that embryo rescue techniques permit the recovery of hybrids from reciprocal crosses between these parents. Most crosses between these two species are successful only when $B$. rapa is the pistillate parent (Olsson and Ellerström, 1980).

Producing somatic hybrids did not require the availability of specific selectable mutant lines (Bravo and Evans, 1985), suspension cultures, or sophisticated instrumentation. Sundberg and Glimelius (1986), in proposing the resynthesis of B. napus as a model system for studying somatic hybrids, relied on experimental procedures involving highly regenerable protoplasts from hypocotyls of one parent fused to leaf cells of the other to permit selection of heterokaryons by micromanipulation or flow sorting. Such a scheme is applicable when abundant seed of one parent is available and when there is access to a micromanipulator or cell sorter, but may be somewhat limited for genetic comparisons of sexual and somatic hybrids due to the destructive nature of obtaining protoplasts from hypocotyls that are subject to variability within seed lots. Since the use of leaf tissue as a source of protoplasts from both parents did not sacrifice the entire plant, our production strategy allowed for continued development of parent plants for concurrent analysis with the somatic hybrids and producing additional hybrids (somatic or sexual) without the contribution of factors that may provide uncontrollable variation. While some somaclonal variation is inherent in the protoplast culture system, the short culture period and prompt hybrid regeneration in our system may minimize its artifactual effects in the hybrid population.

Sexual hybrids between the two progenitor species were produced in both directions, but hybrids were obtained only with one of the two $B$. rapa siblings, whereas both siblings produced somatic hybrids. We find it significant that two siblings contained sufficient genetic differences to prevent the sexual hybridization to $B$. oleracea in one case and not the other. Differences in the selfincompatibility alleles present in the two $B$. rapa plants may account for our inability to produce interspecific sexual hybrids with $B$. rapa-4; the somatic hybrids obtained with this species parents were self-incompatible, whereas those with $B$. rapa-3 were self-compatible (Ozminkowski and Jourdan, 1993a). The selfincompatibility system in $B$. olerace $a$ and $B$. rapa is believed to have developed in the common ancestor to these species, so it is possible that strong incompatibility alleles interfere with hybridization even when the crosses are made in immature flower buds, before the products of self-incompatibility alleles accumulate in the pistil (Nasrallah et al., 1991). The fact that when the selfcompatible parent (B. oleracea) was used as female the efficiency of hybrid formation was nine times greater than when the selfincompatible parent ( $B$. rapa) was used as female suggests a possible influence of the sporophytically determined self-incompatibility in these interspecific crosses. Self-incompatibility has been implicated as a factor in interspecific hybridization between B. campestris and Raphanus sativus L. (Dolstra and Zuidgeest, 1978). However, additional genetic factors associated with proper endosperm and embryo development likely play a role in the success of these crosses. Any such genetic barriers could restrict the germplasm available for sexual hybridization, but less so for somatic hybridizations. The extent of intracellular incongruities in the somatic hybrids may be less deleterious than the intercellular incongruities in the sexual hybrids.

All $F_{1}$ hybrids were produced by fusion of reduced gametes; we did not obtain allotetraploid hybrids directly. Apparently, unreduced gametes are rare in the species parents used here; we did not observe any unusually large pollen grains in these plants, which, in other species have indicated the presence of unreduced gametes. Brassica spp. are known to produce $2 n$ gametes, but the frequency of occurrence is not known (Ollson and Ellerström, 1980). Exploitation of unreduced gametes has been very successful in potato (Solanum tuberosum L.) and in some fruit crops (Peloquin and Ortiz, 1992; Sanford, 1983). Sexual hybridization by unreduced gametes from first division restitution should produce hybrids that are most similar to the somatic hybrids, at least at the level of intragenomic heterozygosity, and one of our goals was to produce such hybrids. Brassica breeders have used tetraploid parents to produce sexual hybrids, but diploid parents may be preferred for practical reasons. Diploidization of the sexual hybrids by colchicine treatment was successful, and we doubled the nuclear DNA complement in eight of the 10 sexual hybrids. The presence of chimeras and highly polyploid plants demonstrates the pitfalls associated with this doubling technique, which requires treating and screening many plants to obtain the desired ones. The procedure we used involved a period of in vitro passage; however, the material used contained preexisting meristems from the embryo and callus was not produced; such conditions are less likely to result in significant somaclonal variation that may complicate analysis of genetic variability in the $\mathrm{F}_{1}$ population. Diploidization was also achieved by protoplast culture of one sexual hybrid, but this method increases the risk of culture-induced variation and is not recommended as a routine polyploidization technique.

An important aspect of these hybridization experiments was the large number of hybrids obtained. Although, in theory, a single somatic hybrid would combine the entire, highly heterozygous nuclear genome of the species parents, it was essential to produce enough hybrids to demonstrate that the plants with balanced genomes displayed uniform characteristics that were derived only from the parents and were not a result of somaclonal variation. A similar rationale justified the need for many $\mathrm{F}_{1}$ hybrids to assess the degree of variability (for hybrids from $n$ gametes) or uniformity (for hybrids from $2 n$ gametes) in the population. Several factors were probably involved in our success in somatic hybrid production, but the principal one was likely the high shoot regeneration ability of purple kale. Such regeneration potential is believed to be under dominant genetic control (Dulieu, 1991; Harms, 1983) and can contribute to successful regeneration of fusion products. A second factor may be the culture system used. Similar culture conditions yielded many somatic hybrids derived from very different genotypes (Jourdan et al., 1989). Using feeder layers (Walters and Earle, 1990) may increase the number of somatic hybrids obtained in similar fusions (Heather and Earle, 1991). Using highly heterozygous fusion partners also may have enhanced the vigor of the fusion products beyond what has been observed in similar attempts at somatic resynthesis of B. napus (Schenck and Röbbelen, 1982; Sundberg and Glimelius 1986). The resulting highly heterozygous somatic hybrids may have exhibited heterosis for growth in vitro, as was demonstrated by the efficient regeneration of plants from leaf protoplasts of the somatic and selected sexual hybrids (unpublished observations). In a similar attempt at resynthesizing $B$. napus by somatic and sexual hybridization of the same parents, Heather and Earle (1991) produced many somatic 
hybrids in five combinations of three $B$. olerace $a$ and three $B$. rapa genotypes, but obtained only one allodiploid sexual hybrid from 90 crosses between 11 genotypes of B. oleracea and 13 of B. rapa, without embryo rescue.

Our production of sexual hybrids also compares favorably with that reported previously. The most efficient method of B. napus resynthesis has been by crosses between tetraploid parents; at the diploid level, the cross is nearly 35 times more efficient with $B$. rapa as the pistillate parent (Olsson and Ellerström, 1980). We obtained 27 hybrids after 550 pollinations, a 5\% efficiency that compares with the maximum $8 \%$ efficiency at the tetraploid level (Olsson and Ellerström, 1980). This success is undoubtedly attributed partly to improved embryo culture procedures and to congruous interactions between the two genomes in the hybrids.

The somatic and sexual hybrids described in this report represent a unique material that can be used to analyze an array of intraand interlocus interactions between the B. olerace $a$ and $B$. rapa genomes combined in the plants. The interactions are likely to be expressed in characteristics such as morphology; growth habit; physiological processes (e.g., response to vernalization); fertility and seed production; and molecular processes such as genome organization, production of fatty acids, glucosynolates, and pigments. Comparing hybrids and their subsequent progeny over many generations may provide insight into the role of intra- and intergenomic heterozygosity in the genetic behavior of these plants for characteristics of agronomic value and botanical interest.

\section{Literature Cited}

Arumuganathan, K. and E.D. Earle. 1991. Estimation of nuclear DNA content of plants by flow cytometry. Plant Mol. Biol. Rpt. 9:229-241.

Bravo, J.E. and D.A. Evans. 1985. Protoplast fusion for crop development, p. 193 220. In: J. Janick (ed.). Plant breeding reviews. AVI Publishing Co., Wesport, Conn.

Chen, B.Y. and W.K. Heneen 1989. Resynthesized Brassica napus L.: A review of its potential in breeding and genetic analysis. Hereditas 111:255-263.

Dolstra, O. and J.H.M. Zuidgeest. 1979. Breeding barriers between Brassica campestris L. and Raphanus sativus L., p. 217-219. In: A.C. Zeven and A.M. van Harten (eds.). Proc. Conf. Broadening Genet. Base Crops 1978, Pudoc, Wageningen.

Dulieu, H. 1991. Inheritance of the regeneration capacity in the genus Petunia. Euphytica 53:173-181.

Glimelius, K. 1985. Sexual and somatic hybridization. Hereditas Suppl. 3:41-47.

Gottlieb, L.D. 1981. Gene numbers in species of Astereae that have different chromosome numbers. Proc. Natl. Acad. Sci. USA 78:3726-3729.

Hadley, H.H. and S.J. Openshaw. 1980. Interspecific and intergeneric hybridization, p. 133-160. In: W.R. Fehr and H.H. Hadley (eds.). Hybridization of crop plants. Amer. Soc. Agron., Madison, Wis.

Harms, C.T. 1983. Somatic incompatibility in the development of higher plant somatic hybrids. Quarterly Rev. Biol. 58:325-353.

Heather, D.W. and E.D. Earle. 1991. Resynthesis of novel rapeseed via protoplast fusion, p. 1090-1095. In: D.I. McGregor (ed.). Proc. 8th Intl. Rapeseed Congr. Organizing Committee, Agriculture Canada, Saskatoon.

Hebert, P.D.N. and M.J. Beaton. 1989. Methodologies for allozyme analysis using cellulose acetate electrophoresis: A practical handbook. Helena Laboratories, Beaumont, Texas.

Inomata, N. 1978. Production of interspecific hybrids in Brassica campestris $\mathrm{x} B$. oleracea by culture in vitro of excised ovaries. I. Development of excised ovaries in the crosses of various cultivars. Jpn. J. Genet. 53:161-173.

Jourdan, P.S. and E.D. Earle. 1989. Genotypic variability in the frequency of plant regeneration from leaf protoplasts of four Brassica spp. and of Raphanus sativus. J. Amer. Soc. Hort. Sci. 114:343-349.

Jourdan, P.S., E.D. Earle, and M.A. Mutschler. 1989. Synthesis of male sterile, triazine-resistant Brassica napus by somatic hybridization between cytoplasmic male sterile B. olerace a and atrazine-resistant B. campestris. Theoretical Applied Genet. 78:445-455.
Kräling, K. 1987. Utilization of genetic variability of resynthesized rapeseed. Plant Breeding 99:209-217.

Kumar, A. and E.C. Cocking. 1987. Protoplast fusion: A novel approach to organelle genetics in higher plants. Amer. J. Bot. 74:1289-1303.

McNaughton, I. and C.L. Ross. 1978. Annual report of the Scottish plant breeding station. Invergowrie, Scotland. p. 75-110.

Murashige, T. and F. Skoog. 1962. A revised medium for rapid growth and bioassays with tobacco tissue cultures. Physiol. Plant. 15:473-497

Nasrallah, J.B., T. Nishio, and M.E. Nasrallah. 1991. The self-incompatibility genes of Brassica: Expression and use in genetic ablation of floral tissues. Annu. Rev. Plant Physiol. Plant Mol. Biol. 42:393-422.

Negrutiu, I., S. Hinnisdaels, A. Mouras, B.S. Gill, G.B. Gharti-Chhetri, M.R. Davey, Y.Y. Gleba, V. Sidorov, and M. Jacobs. 1989. Somatic versus sexual hybridization: Features, facts and future. Acta Bot. Neerl. 38:253-272.

Nishi, S. 1980. Differentiation of Brassica crops in Asia and the breeding of 'Hakuran,' a newly synthesized leafy vegetable, p. 133-150. In: S. Tsunoda, K. Hinata, and C. Gómez-Campo (eds.). Brassica crops and wild allies. Jpn. Sci. Soc. Press, Tokyo.

Olsson, G. 1960. Species crosses within the genus Brassica. II. Artificial Brassica napus L. Hereditas 46:351-386.

Olsson, G. and S. Ellerström. 1980. Polyploidy breeding in Europe, p. 167-190. In: S. Tsunoda, K. Hinata, and C. Gómez-Campo (eds.). Brassica crops and wild allies. Jpn. Sci. Soc. Press, Tokyo.

Ozminkowski, Jr., R.H. and P.S. Jourdan. 1993a. Expression of self-incompatibility and fertility of Brassica napus resynthesized by interspecific somatic hybridization. Euphytica 65:153-160.

Ozminkowski, Jr., R.H. and P.S. Jourdan. 1993b. Compariing the resynthesis of Brassica napus by interspecific somatic and sexual hybridization. II. Hybrid morphology and identifying organelle genomes. J. Amer. Soc. Hort. Sci. 119:816823.

Pelletier, G., C. Primard, F. Vedel, P. Chetrit, R. Remy, P. Rousselle, and M. Renard. 1983. Intergeneric cytoplasmic hybridization in Cruciferae by protoplast fusion. Mol. Gen. Genet. 191:244-250.

Peloquin S.J. and R. Ortiz. 1992. Techniques for introgressing unadapted germplasm to breeding populations, p. 485-507. In: H.T. Stalker and J.P. Murphy (eds.). Plant breeding in the 1990s. CAB International, Wallingford, U.K.

Quazi, M.H. 1988. Interspecific hybrids between Brassica napus L. and B. oleracea L. developed by embryo culture. Theoretical Applied Genet. 75:309318.

Ripley, V.L. and P.G. Arnison. 1990. Hybridization of Sinapis alba L. and Brassica napus L. via embryo rescue. Plant Breeding 104:26-33.

Robertson, D., J.D. Palmer, E.D. Earle, and M.A. Mutschler. 1987. Analysis of organelle genomes in a somatic hybrid derived from cytoplasmic male-sterile Brassica oleracea and atrazine-resistant B. campestris. Theoretical Applied Genet. 74:303-309.

Sanford. J.C. 1983. Ploidy manipulations, p. 100-123. In: J.N. Moore and J. Janick (eds.). Methods in fruit breeding. Purdue Univ. Press, West Lafayette, Ind.

Schenck, H.R. and G. Röbbelen. 1982. Somatic hybrids by fusion of protoplasts from Brassica oleracea and B. campestris. Z. Pflanzenzücht. 89:278-288.

Song, K.M., T.C. Osborn, and P.H. Williams. 1988. Brassica taxonomy based on nuclear restriction fragment length polymorphisms. I. Genome evolution of diploid and amphidiploid species. Theoretical Applied Genet. 75:784-794.

Sundberg, E. and K. Glimelius. 1986. A method for the production of interspecific hybrids within Brassiceae via somatic hybridization, using resynthesis of Brassica napus as a model. Plant Sci. 43:155-162.

Sundberg, E., M. Landgren, and K. Glimelius. 1987. Fertility and chromosome stability in Brassica napus resynthesized be protoplast fusion. Theoretical Applied Genet. 75:96-104.

Sybenga, J. 1983. Genetic manipulation in plant breeding: Somatic versus generative. Theoretical Applied Genet. 66:179-201.

Terada, R., Y. Yamashita, S. Nishibayashi, and K. Shimamoto. 1987. Somatic hybrids between Brassica oleracea and B. campestris: Selection by the use of iodoacetamide inactivation and regeneration ability. Theoretical Applied Genet. 73:379-384.

Thorpe, M.L., L.H. Duke, and W.D. Beversdorf. 1987. Procedures for the detection of isozymes of rapeseed by starch gel electrophoresis. Agr. Can. Tech. Bul. OAC 887, Univ. of Guelph, Ontario, Canada.

Waters, T.W. and E.D. Earle. 1990. A simple, versatile feeder layer system for Brassica oleracea protoplast culture. Plant Cell Rpt. 9:316-319. 